\title{
Steven Johnson Syndrome Due to Allopurinol Use
}

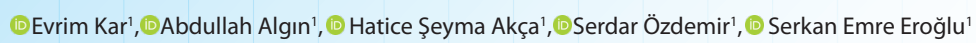

'Department of Emergency Medicine, Health Sciences University, Ümraniye Education and Research Hospital, Istanbul, Turkey.

\begin{abstract}
Steven Johnson syndrome is a severe cutaneous adverse reaction that develops especially against drugs and has an increasing incidence. The mortality rate in the elderly population is quite high compared to other age groups. Rapid diagnosis, early recognition, and discontinuation of the responsible drug reduce the mortality rate in patients admitted to the emergency department. The most important step in treatment is supportive treatment.

We present a 70-year-old woman with a history of hypertension, coronary artery disease, known renal failure, and adrenal insufficiency. Steven Johnson syndrome developed. We emphasized that the use of allopurinol due to known renal failure and hyperuricemia increased the mortality rate by causing acute kidney damage, and after its rapid diagnosis and treatment, it positively affected mortality. The patient was admitted to the internal medicine service with a pre-diagnosis of Steven Johnson syndrome in the emergency department and was discharged with recommendations after 26 days of hospitalization.

Because of the rapid spread and rapid deterioration of the general condition in severe cutaneous reactions such as Steven Johnson, mortality is important to make a rapid diagnosis, to determine the etiology, and to start treatment early.

Keywords: steven johnson syndrome, allopurinol, drug adverse effect.
\end{abstract}

\section{Introduction}

Stevens-Johnson syndrome (SJS) and toxic epidermal necrolysis (TEN) are diseases mentioned together in the literature and are severe cutaneous adverse reactions characterized by extensive separation of the epidermis and mucous membrane erosions ${ }^{1}$. The most basic feature that distinguishes SJS from TEN is that it goes with less than $10 \%$ body surface area involvement, with an annual incidence of $1.5-1.8 / 1.000 .000^{2}$; It is considered a small form of toxic epidermal necrolysis with an average mortality rate of $1-5 \%{ }^{3}$. At least $50 \%$ of the cases occur due to drugs ${ }^{1}$. Various antiepileptic drugs, sulfonamide antibiotics, allopurinol, antiretroviral drugs and oxicam analgesics have been identified as triggers of $\mathrm{SJS}^{4}$.

The clinic begins with fever, sore throat, runny nose, and myalgia. Subsequently, painful rash lesions that spread rapidly occur. The prognosis depends on the degree of skin peeling and the development of secondary bacterial infections. In uncomplicated cases, lesions heal within 1-2 weeks without any sequelae ${ }^{3}$. The first- and third-day measurements named "scorten", which consists of age, malignancy, blood urea nitrogen, glucose, pulse, serum bicarbonate level, and body level, developed by Bastuji-Garin et al. used $a^{5,6}$ Identifying the causative drug and discontinuing it early is very important for the survival of patients with SJS 7 . This situation shows the importance of rapid clinical diagnosis in patients admitted to the emergency department.

In this case report, it is aimed to present a patient with SJScaused by allopurinol and discuss it in the light of current literature.

\section{Case Report}

A 74-year-old female patient was admitted to the emergency department with the complaint of diffuse maculopapular erythematous, itchy rashes that started from the trunk, back and progressed to the palmar faces of the hands and feet, and swallowing difficulties, keeping less than $10 \%$ of the total body surface area. It was learned that the patient, who did not have a history of allergy, used allopurinol, which was initiated 14 days ago due to hyperuricemia, amlodipine $10 \mathrm{mg}$ for hypertension, atorvastatin $40 \mathrm{mg}$ for coronary artery disease and hyperlipidemia, and warfarin $5 \mathrm{mg}$ due to a history of arrhythmia. On physical examination, his general condition was moderate, and his GlasgowComa Scale was 14. In vital parameter analysis; blood pressure: 100/50 $\mathrm{mmHg}, \mathrm{SaO}_{2}$ : $97 \%$, heart rate: 95 /minute, fever: $37.8^{\circ} \mathrm{C}$. In the tachypneic patient, bilateral respiratory sounds were

Corresponding Author: Evrim Kar e-mail: evrmkar@gmail.com 
Table 1. Initial Laboratory Findings of Patient

\begin{tabular}{ll}
\hline Albumin: $23 \mathrm{~g} / \mathrm{dL}$ & Lipase: $42 \mathrm{U} / \mathrm{L}$ \\
Leukocyte: $3.59 \mathrm{u} / \mathrm{L}$ & Amylase: $88 \mathrm{U} / \mathrm{L}$ \\
Neutrophil: $2.15 \mathrm{U} / \mathrm{L}$ & Gamma glutamyl transferase: $203 \mathrm{U} / \mathrm{L}$ \\
Eosinophil: $0 \mathrm{U} / \mathrm{L}$ & Sodium: $132 \mathrm{mEq} / \mathrm{L}$ \\
Hemoglobin: $10.5 \mathrm{~g} / \mathrm{dL}$ & Potassium: $4.3 \mathrm{mEq} / \mathrm{L}$ \\
Hematocrit: $31.2 \%$ & Chlorine: $114 \mathrm{mEq} / \mathrm{L}$ \\
Platelet: $116 \mathrm{U} / \mathrm{L}$ & Calcium: $6.7 \mathrm{mg} / \mathrm{dL}$ \\
Creatinine: $1.89 \mathrm{mg} / \mathrm{dL}$ & Corrected Calcium: 8 \\
Blood urea nitrogen: $143 \mathrm{mg} / \mathrm{dL}$ & C-Reactive Protein: $3.9 \mathrm{mg} / \mathrm{dL}$ \\
Uric acid: $10.5 \mathrm{mg} / \mathrm{dL}$ & $\mathrm{pH}^{2} 7.27$ \\
INR: 1.32 & $\mathrm{pCO}_{2}: 29.8 \mathrm{mmHg}$ \\
Prothrombin time: $17.4 \mathrm{~seconds}$ & $\mathrm{HCO}_{3}: 13.5 \mathrm{mmol} / \mathrm{L}$ \\
Lactate dehydrogenase: $462 \mathrm{U} / \mathrm{L}$, & Lactate: $1.9 \mathrm{mmol} / \mathrm{L}$ \\
Alanine transaminase: $38 \mathrm{U} / \mathrm{L}$ & Base minus: $-11.9 \mathrm{mmol} / \mathrm{L}$
\end{tabular}

Aspartate transaminase: $52 \mathrm{U} / \mathrm{L}$

decreased, and basal rales were present. There was minimal tenderness in the abdomen. There were dry oropharynx, hemorrhagic ulcerated crusts on the oral mucosa and widespread erythematous squamous rashes on the body. The Nikolsky sign was positive.

There was no abnormality other than atrial fibrillation on electrocardiography. Initial laboratory findings are shown in table 1.

Patient was hospitalized with diagnosis of SJS secondary to allopurinol.In medical treatment allopurinol was stopped, fluid replacement planned, and broad-spectrum antibiotics as ciprofloxacin $400 \mathrm{mg}$ twice a dayfor 5 days and deltacortril5 mg once a day for 5 days was started. During follow-up, growth of methicillin-resistant Staphylococcus aureus in blood culture and growth of Pseudomonas and Enterobacter in urine culture were observed. Daptomycin 1 x $10 \mathrm{mg} / \mathrm{kg} /$ day and teicoplanin $400 \mathrm{mg}$ twice a dayas loading dose and $400 \mathrm{mg}$ once a day as maintenance dose were started orally. The patient's initial SCORTEN scale value was 3, and the probable mortality rate was 35.3 . Although no examination regarding the histopathology results of the skin biopsy for the definitive diagnosis of the disease was obtained, the clinical diagnosis was a priority disease and the patient received a response to the treatment, even the SCORTEN scale value was 2 on the $3^{\text {rd }}$ day and the possible mortality rate decreased to 12.1 . The patient was discharged on the $26^{\text {th }}$ day without any complication.

\section{Discussion}

SJS is a rare severe epidermolysis adverse cutaneous reaction associated with drug use $\mathrm{p}^{7}$. Its frequency is higher in women than men, and it occurs at all ages, but its frequency increases with age ${ }^{8}$. In our study, we aimed to present a case in which squamous erythematous rashes on the skin of an elderly female patient, thought to be secondary to drug reaction, regressed with allopurinol discontinuation.

Allopurinol is used to reduce serum uric acid levels in patients with gout and hyperuricemia ${ }^{9}$. Although the clinical process is usually within 1-3 weeks after drug intake, the disease usually occurs within the first 2 months ${ }^{10}$. In our case, there was a clinical course with painful lesions with rash after fever and myalgia, which occurred after the use of allopurinol 14 days ago. In a retrospective cohort study by Kim et al. in which 65,625 patients using allopurinol, 45 of whom were hospitalized for severe, cutaneous adverse reactions, using the drug data analysis system, the risk of developing SJS in allopurinol users was 10 times higher than in those who did not use allopurinol. found that ${ }^{11}$. Frey et al. found that the risk of SJS increased in allopurinol users in a case-control study created by matching SJS patients with and without allopurinol in a 1: 4 manner, which was previously validated by SJS ${ }^{12}$.

Although adverse reactions seen with allopurinol are rare, the mortality rate of SJS cases caused by allopurinol 
is $25 \% .^{13}$ Various scoring systems are used to predict mortality in patients diagnosed with SJS. SCORTEN, ALDEN, ICNARC, APACHE are some of the scales used in predicting mortality. In a study by Lerch et al. Dated 2018, they concluded that the "SCORTEN" mortality severity scale in SJS is superior to other scoring systems such as ALDEN, APACHE II and ICNARC ${ }^{6}$ In our patient, the SCORTEN scale was used to predict mortality, and the initial mortality While the rate was $35.3 \%$, the 3 -day mortality rate was $12.1 \%$.

Sepsis and multiple organ failure are the most important causes of death ${ }^{14}$. The aim of treatment is to prevent the development of these complications. The most important step is early diagnosis and discontinuation of suspected drug or drugs. Providing fluid and electrolyte balance and nutritional support, protection from infection, respiratory support, adjustment of external temperature, pain and anxiety management, skin and wound care are the other steps of treatment ${ }^{15}$. Our patient was discharged after 26 days with drug discontinuation and fluid, antibiotics, and supportive therapy.

As a result; although we do not deal with the diseases with skin reaction in the emergency service as seriously as the care of other patients with hemodynamic instability, we think that this case report will create an important awareness in the literature on behalf of the emergency department management perspective in order to recognize patients with such drug reactions and with high mortality.

\section{References}

1. Roujeau JC, Stern RS. Severe Adverse Cutaneous Reactions to Drugs. N Engl J Med. 1994;331(19):1272-85.

2. Paulmann M, Mockenhaupt M. Schwere arzneimittelinduzierte Hautreaktionen: Klinik, Diagnostik, Ätiologie und Therapie. JDDG - J Ger Soc Dermatology. 2015;13(7):625-43.

3. Schneider JA, Cohen PR. Stevens-Johnson Syndrome and Toxic Epidermal Necrolysis: A Concise Review with a Comprehensive Summary of Therapeutic Interventions Emphasizing Supportive Measures. Adv Ther. 2017;34(6):1235-44.

4. Mockenhaupt M, Viboud C, Dunant A, Naldi L, Halevy S, Bavinck JNB, et al. Stevens-Johnson syndrome and toxic epidermal necrolysis: Assessment of medication risks with emphasis on recently marketed drugs. The EuroSCAR-study. J Invest Dermatol. 2008;128(1):35-44.
5. Bastuji-Garin S, Fouchard N, Bertocchi M, Roujeau JC, Revuz J, Wolkenstein P. Scorten: A severity-of-illness score for toxic epidermal necrolysis. J Invest Dermatol. 2000;115(2):149-53.

6. Lerch M, Mainetti C, Terziroli Beretta-Piccoli B, Harr T. Current Perspectives on Stevens-Johnson Syndrome and Toxic Epidermal Necrolysis. Clin Rev Allergy Immunol. 2018;54(1):147-76.

7. Harr T, French LE. Stevens-Johnson syndrome and toxic epider- mal necrolysis. Chem Immunol Allergy. 2012 Jan;97:149-66.

8. Roujeau JC, Guillaume JC, Fabre JP, Penso D, Fléchet ML, Girre JP.Toxic epidermal necrolysis (Lyell syndrome). Incidence and drug etiology in France, 19811985. Arch Dermatol 1990;126:37-42.

9. Ramasamy SN, Korb-Wells CS, Kannangara DR, Smith M,WangN, Roberts D et al. (2103) Allopurinol hypersensitivity: a systematic review of all published cases, 1950-2012. Drug Saf36, 953-80

10. Mockenhaupt M. Severe drug-induced skin reactions: clinical pattern, diagnostics and therapy. J Dtsch Dermatol Ges. 2009;7:142-60.

11. Kim SC, Newcomb C, Margolis D,RoyJ,Hennessy S. Severe cutaneous reactions requiring hospitalization in allopurinol initiators: a population-based cohort study. Arthritis Care Res. 2013;65(4):578-584.)

12. Frey $N$, Bodmer $M$, Bircher $A$, Jick SS, Meier CR, Spoendlin J. Stevens-Johnson Syndrome and Toxic Epidermal Necrolysis in Association with Commonly Prescribed Drugs in Outpatient Care Other than Anti-Epileptic Drugs and Antibiotics: A Population-Based Case-Control Study. Drug Saf [Internet]. 2019;42(1):55-66. Available from: https://doi.org/10.1007/s40264-018-0711-x

13. Saito $Y$, Stamp LK, Caudle KE, Hershfield MS, McdonaghEM,Callaghan JT. (2016) Clinical Pharmacogenetics Implementation Consortium (CPIC) guide- lines for human leukocyte antigen B (HLA-B) genotype and allopurinol dosing: 2015 update. Clin Pharmacol Ther 99, 36-7.

14. Ghislain PD , Roujeau JC: Treatment of severe drug reactions: Stevens-Johnson syndrome, toxic epidermal necrolysis and hyper- sensitivity syndrome. Dermatol Online J 2002;8:5.)

15. Chave TA, Mortimer NJ, Sladden MJ, Hall AP, Hutchinson PE: Toxic epidermal necrolysis: current evidence, practical management and future directions. Br J Dermatol 2005;153:241-53. 